\title{
Comunicação
}

[Communication]

\section{Anticorpos contra lentivírus de pequenos ruminantes em caprinos e ovinos em abatedouros do estado de Pernambuco}

[Small ruminant lentivirus infection in goats and sheep from two abattoirs in Pernambuco State, Brazil]

\author{
M.M.M. Oliveira ${ }^{1,4}$, R.S. Castro ${ }^{1 *}{ }^{*}$, K.L. Carneiro $^{2}$, S.A. Nascimento ${ }^{1}$, \\ A.K.C. Callado ${ }^{3}$, C.S.A. Alencar ${ }^{3}$, L.S.P. Costa ${ }^{1}$ \\ ${ }^{1}$ Departamento de Medicina Veterinária - UFRPE \\ Rua Dom Manoel de Medeiros, s/n., Dois Irmãos \\ 52171-900 - Recife, PE \\ ${ }^{2}$ Médico Veterinário \\ ${ }^{3}$ Laboratório de Apoio Animal - Recife, PE \\ ${ }^{4}$ Bolsista da CAPES
}

Os lentivirus de pequenos ruminantes (LVPR CAEV e Maedi-Visna), que acometem caprinos e ovinos, pertencem à família Retroviridae e subfamília Lentivirinae (International.... 2004) e caracterizam-se por apresentarem período de incubação longo, variando de meses a anos, evolução geralmente crônica, com agravamento progressivo das lesões, perda de peso e debilidade até a morte (Narayan e Cork, 1985; Callado, et al., 2001).

No Brasil, vários relatos indicam que os LVPR estão presentes em vários estados. Em Pernambuco, estudos epidemiológicos têm demonstrado prevalência de aproximadamente $17,0 \%$ em animais de raças especializadas para produção de leite (Castro et al., 1994), porém não existem relatos sobre a prevalência dessa virose em rebanhos caprinos sem raça definida (SRD), criados tradicionalmente para produção de carne e pele (Castro, 1999). Inquéritos sorológicos sobre esses vírus em ovinos não foram ainda realizados, estando a atual situação do problema indefinida. O objetivo deste trabalho é apresentar os resultados da prevalência de LVPR em caprinos e ovinos SRD, enviados para o abate.
Colheram-se amostras de dois abatedouros, localizados nos municípios de São Lourenço da Mata e Paulista, do estado de Pernambuco, no período de novembro de 1999 a março de 2001.

Amostras de sangue foram coletadas dos animais disponíveis para o abate, independente de espécie, sexo e idade. A idade dos animais foi estimada com base no número de mudas de dentes. $\mathrm{O}$ número necessário de amostras colhidas para estimativa da prevalência foi determinado utilizando-se o programa Epi-Info (Dean et al.,1994), que emprega a fórmula apresentada por Kish (1965), para estudo populacional, por amostragem aleatória.

Para detecção dos anticorpos anti-LVPR, foi utilizado o teste de imunodifusão em gel de ágar (IDGA), com antígeno produzido a partir de sobrenadantes de células de membrana sinovial ovina (MSO), infectadas com MVV amostra K1514 (Abreu et al., 1998). O soro padrão positivo foi obtido de um animal forte positivo, purificado utilizando-se sulfato de amônia (Hudson e Hay, 1980). Para preparação do gel, foi utilizada agarose $1 \%(\mathrm{p} / \mathrm{v})$ em solução tampão borato de sódio (108 mM; pH 8,6). Os dados obtidos com

Recebido para publicação em 27 de maio de 2004 Aceito em 4 de novembro de 2005

*Autor para correspondência (corresponding author)

E-mail: rscastro@ufrpe.br

Apoio $\mathrm{CNPq}$ 
o teste dessas amostras foram submetidos ao teste de qui-quadrado $\left(\chi^{2}\right)$ e à prova exata de Fisher, com o auxílio do Epi-Info (Dean et al.,1994).

Das 997 amostras de soro colhidas, 672 foram de caprinos e 325 de ovinos. Pelo IDGA determinou-se a prevalência de $3,8 \%$ $(3,0 \leq \mathrm{P} \leq 4,5)$ e $5,2 \%(4,0 \leq \mathrm{P} \leq 6,5)$ de caprinos e ovinos, respectivamente, portadores de anticorpos precipitantes contra LVPR. No abatedouro de São Lourenço da Mata, foram colhidas 476 amostras de soro caprino, com $3,2 \%(2,7 \leq \mathrm{P} \leq 4,0)$ de resultados positivos; das 227 amostras de soro ovino, 4,0\% $(2,7 \leq \mathrm{P} \leq 5,3)$ foram positivas. No abatedouro de Paulista, 5,1\% (10/196) $(3,5 \leq \mathrm{P} \leq 6,7)$ dos caprinos amostrados apresentaram resultado positivo, e $8,2 \%(8 / 98)$ $(5,4 \leq \mathrm{P} \leq 11,0)$ dos ovinos foram soropositivos (Tab. 1 e 2). Observou-se, ainda, que não houve diferença significativa entre os abatedouros, quanto à positividade para $\operatorname{LVPR}\left(\chi^{2} ; \mathrm{P}>0,05\right)$.

Tabela 1. Resultado do teste de imunodifusão em gel de ágar de caprinos sem raça definida, de acordo com a localização do abatedouro, sexo e faixa etária, para pesquisa de anticorpos contra lentivírus de pequenos ruminantes, amostrados no período de novembro de 1999 a março de 2001

\begin{tabular}{|c|c|c|c|c|}
\hline Estrato & & Negativo (\%) & Positivo (\%) & Total de amostras (\%) \\
\hline Localização do abatedouro & $\begin{array}{c}\text { São Lourenço da } \text { Mata }^{1} \\
\text { Paulista }^{1}\end{array}$ & $\begin{array}{l}461(96,85) \\
186(94,89)\end{array}$ & $\begin{array}{l}15(3,15 \pm 0,80) \\
10(5,10 \pm 1,57)\end{array}$ & $\begin{array}{l}476(70,83) \\
196(29,17)\end{array}$ \\
\hline Sexo & $\begin{array}{l}\text { Macho }^{2} \\
\text { Fêmea }^{2}\end{array}$ & $\begin{array}{l}410(95,79) \\
237(97,13)\end{array}$ & $\begin{array}{c}18(4,20 \pm 0,97) \\
7(2,87 \pm 0,11)\end{array}$ & $\begin{array}{l}428(63,39) \\
244(36,31)\end{array}$ \\
\hline Número de mudas (idade) & $\begin{array}{l}\text { Até } 1^{3} \\
2 \text { a } 3^{3} \\
>4^{3}\end{array}$ & $\begin{array}{c}60(93,75) \\
194(98,47) \\
393(95,62)\end{array}$ & $\begin{array}{c}4(6,25 \pm 3,02) \\
3(1,52 \pm 0,87) \\
18(4,38 \pm 1,01)\end{array}$ & $\begin{array}{c}64(9,25) \\
197(29,32) \\
411(61,16)\end{array}$ \\
\hline
\end{tabular}

${ }^{1}\left(\chi^{2}=2,14 ; \mathrm{P}>0,05\right)$

${ }^{2}\left(\chi^{2}=0,45 ; \mathrm{P}>0,05\right)$

${ }^{3}\left(\chi^{2}=4,30 ; \mathrm{P}>0,05\right)$; número de mudas corresponde a faixa etária em meses (até $1-$ de 0 a 24 meses; 2 a 3 - 24 a 48 meses; > 4 acima de 48 meses).

Tabela 2. Resultado do teste de imunodifusão em gel de ágar de ovinos sem raça definida, de acordo com a localização do abatedouro, sexo e faixa etária, para pesquisa de anticorpos contra lentivírus de pequenos ruminantes, amostrados no período de novembro de 1999 a março de 2001

\begin{tabular}{lcccc}
\hline Estrato & & Negativo (\%) & Positivo (\%) & $\begin{array}{c}\text { Total de amostras } \\
(\%)\end{array}$ \\
\hline Localização do abatedouro & $\begin{array}{c}\text { São Lourenço da Mata } \\
\text { Paulista }^{1}\end{array}$ & $218(96,03)$ & $9(3,96 \pm 1,29)$ & $227(69,84)$ \\
& Macho $^{2}$ & $233(93,57)$ & $16(6,42 \pm 1,55)$ & $249(76,62)$ \\
Sexo & Fêmea $^{2}$ & $75(98,68)$ & $1(1,32 \pm 1,31)$ & $76(23,38)$ \\
& Até 1 $^{3}$ & $33(100,00)$ & --- & $33(10,15)$ \\
Número de mudas (idade) & 2 a 3 $^{3}$ & $62(19,08)$ & $2(3,13 \pm 2,18)$ & $64(19,69)$ \\
& $>4^{3}$ & $213(93,42)$ & $15(6,58 \pm 1,64)$ & $228(70,16)$ \\
\hline
\end{tabular}

${ }^{1}\left(\chi^{2}=2,43 ; \mathrm{P}>0,05\right)$

2 (Teste exato de Fisher; $\mathrm{P}>0,05$ )

${ }^{3}\left(\chi^{2}=3,23 ; \mathrm{P}>0,05\right)$; número de mudas corresponde a faixa etária em meses (até $1-$ de 0 a 24 meses; 2 a 3 - 24 a 48 meses; $>4-$ acima de 48 meses).

Do total de caprinos amostrados, 4,2\% $\pm 0,97$ correspondiam a animais machos soropositivos e $2,9 \pm 0,11$ a fêmeas soropositivas. No caso dos ovinos, $6,4 \% \pm 1,55$ e $1,3 \% \pm 1,31$ dos resultados positivos foram de machos e fêmeas soropositivos, respectivamente. Não houve diferenças entre os sexos quanto à positividade para LVPR $\left(\chi^{2} ; \mathrm{P}>0,05\right)$. A distribuição dos elementos amostrais nesse estrato não condiz com a realidade dos rebanhos, pois, predominantemente, esses são formados por fêmeas. 
A idade prevalente foi acima de 48 meses. Não foram encontradas diferenças, quanto à positividade, de acordo com a faixa etária, em ambas as espécies estudadas $\left(\chi^{2} ; \mathrm{P}>0,05\right)$.

Embora os resultados confirmem a ocorrência de LVPR, devem ser interpretados com cautela, quando se pretende inferir sobre a prevalência real da infecção por LVPR na população caprina e ovina SRD do estado. Inicialmente, sabe-se que é possível haver superestimativa dos resultados positivos quando a prevalência real é baixa, dependendo da sensibilidade do teste utilizado.
Neste trabalho, visando minimizar essa possibilidade, utilizou-se um teste que apresenta alto valor preditivo positivo, diminuindo o "peso" dos resultados falso-positivos no cálculo da prevalência estimada (Thiry e Pastoret, 1992).

Pode-se concluir que os LVPR ocorrem nos rebanhos caprino e ovino SRD do estado de Pernambuco em baixa prevalência.

Palavras-chave: caprino, bovino, CAEV, MaediVisna, epidemiologia

\begin{abstract}
The prevalence of small ruminant lentivirus (SRL) infection was evaluated in goats and sheep in two counties of Pernambuco State, Brasil. Seriological examinations were performed from a total of 672 goats and 325 sheep, one year of age and older, at two abattoirs. Sera were analyzed by agar gel immunodiffusion using Maedi-Visna K-1514 antigens. There were 42 reactive samples (95\% confidence interval 3.6\% to 4.9\%) in both slaughter houses. In São Lourenço da Mata county, 3.2\% and $4.0 \%$ of goat and sheep sera were responsive, whereas in Paulista county, 5.1\% of goat samples and $8.2 \%$ of sheep were reactive. Thus, the prevalence of small ruminant lentivirus was low in goats and sheep sampled from the region.
\end{abstract}

Keywords: goat, sheep, CAEV, Maedi-visna, epidemiology

\section{REFERÊNCIAS BIBLIOGRÁFICAS}

ABREU, S.R.O.; CASTRO, R.S.; NASCIMENTO, S.A. et al. Produção de antígeno nucleoproteíco do vírus da Artrite-Encefalite Caprina e comparação com o do vírus Maedi - Visna para utilização em teste de imunodifusão em agar gel. Pesqui. Vet. Bras., v.18, p.57-60, 1998

CALLADO, A.K.C.; CASTRO, R.S.; TEIXEIRA, M.F.S. Lentivírus de Pequenos Ruminantes (CAEV e Maedi-Visna): Revisão e perspectivas. Pesqui. Vet. Bras., v.21, p.87-97, 2001.

CASTRO, R.S. Principais doenças infecciosas de caprinos. In: CONGRESSO PERNAMBUCANO DE MEDICINA VETERINÁRIA, 4.; SEMINÁRIO NORDESTINO DE CAPRINOOVINOCULTURA, 5., 1999, Recife. Resumo... SPEMVE, 1999. p.116-117.

CASTRO, R.S.; NASCIMENTO, S.A.; ABREU, S.R.O. Evidência sorológica da infecção pelo vírus da artrite-encefalite caprina em caprinos leiteiros no Estado de Pernambuco. Arq. Bras. Med. Vet. Zootec., v.46, p.571-572, 1994.
DEAN, A.G.; DEAN, J.A.; COULOMBIER, D. et al. Epi info. Version 6: a word processing database, and statistics program for epidemiology on microcomputers. Atlanta, Georgia, USA: Center of Disease Control and Prevention, 1994. 589p.

HUDSON, L.; HAY, F.C. Pratical immunology. London: Blackwell Sci., 1980. 340p.,

INTERNATIONAL Committee on Taxonomy of Viruses, 2004. Disponível em: $<$ www.virustaxonomyonline.com $>$. Acessado em: $03 / 09 / 2004$

KISH, L. Survey Sampling. New York: John Wiley \& Sons, 1965.

NARAYAN, O.; CORK, L.C. Lentiviral diseases of sheep and goats: Chronic pneumonia, leukoencephalomyelitis and arthritis. Rev. Infect. Dis., v.7, p.89-97, 1985.

THIRY, E.; PASTORET, P.P. L'évalution des méthodes diagnostiques. Ann. Med. Vet. v.136, p.269-272, 1992. 